\title{
Perfil psicosocial de la mujer emprendedora. Un análisis cualitativo y local
}

DOI: https://doi.org/10.21158/01208160.n88.2020.2697

\author{
Yamila Fernanda Silva-Peralta ${ }^{1}$ \\ Universidad Nacional de Mar del Plata \\ yamilasilvaperalta@gmail.com \\ María Emilia Rompato ${ }^{2}$ \\ Universidad Nacional de Mar del Plata \\ merompato@gmail.com
}

\section{Fecha de recepción: 15 de marzo de 2019}

Fecha de aprobación: 18 de junio de 2020

Cómo citar este artículo / To reference this article / Comment citer cet article / Para citar este artigo:

Silva-Peralta, Y. F.; Rompato, M. E. (2020). Perfil psicosocial de la mujer emprendedora. Un análisis cualitativo y local. Revista Escuela de Administración de Negocios, (88), 145-164. DOI: https://doi.org/10.21158/01208160.n88.2020.2697

\section{Resumen}

Mediante la perspectiva de la motivación inicial, la teoría clásica de las necesidades y el modelo de valores individualistas y colectivistas, se analiza el perfil psicosocial de la mujer emprendedora de Mar del Plata, Argentina. Con una base etnometodológica, se administraron cuestionarios sociodemográficos y entrevistas en profundidad según muestreo intencional, con un $\mathrm{N}=34$. Se realizó análisis de contenido de las respuestas en conformidad con los mencionados modelos teóricos. Las recurrencias muestran que las mujeres desarrollan su emprendimiento con una marcada implicación personal y ante situaciones de criticidad personales o laborales. Así, emprenden tanto por necesidad como por oportunidad, e incorporan una mirada social de especial atención hacia el otro. Desarrollan una característica necesidad de logro, asociada a la superación de obstáculos y el alcance de metas propuestas, aunque también se encuentra necesidad de afiliación, la cual se relaciona con la mirada social antes nombrada. Por último, se han encontrado una conjunción de valores colectivistas asociados a la benevolencia, así como mixtos, asociados al universalismo. Los resultados encontrados, así como su discusión, abren un abanico de preguntas $\mathrm{y}$, a su vez, de posibilidades. El análisis permite concluir que es necesario cuestionarse el espacio de la mujer en el ámbito emprendedor y su necesidad de visibilización.

Palabras clave: emprendimiento; emprendimiento femenino; mujeres emprendedoras; actividad emprendedora; emprendimiento y género; emprendimiento en Argentina; estudio sociodemográfico.

\footnotetext{
${ }^{1}$ Licenciada en Psicología por la Universidad Nacional de Mar del Plata, Argentina. Magíster en Ciencias Humanas y Sociales por la Universidad Rene Descartes Paris 5, Francia. Doctora en Psicología por la Universidad de Barcelona, España. ORCID: https://orcid. org/0000-0003-1917-3406

${ }^{2}$ Licenciada en Sociología por la Universidad Nacional de Mar del Plata, Argentina. Doctoranda en Psicología de la Universidad Nacional de Mar del Plata, Argentina. ORCID: https://orcid.org/0000-0001-6089-2387
} 


\title{
Psychosocial profile of the entrepreneurial woman. A qualitative and local analysis
}

\begin{abstract}
It is by means of the initial motivation perspective, the classic theory of needs, and the model of individualistic and collectivistic values, that the psychosocial profile of the entrepreneurial woman in Mar del Plata, Argentina, is analyzed. On an ethnomethodological basis, we conducted sociodemographic questionnaires and in-depth interviews according to intentional sampling, with $\mathrm{N}=34$, and carried out an analysis of the content of the replies in accordance with the aforementioned theoretical models. The recurrences show that women develop their entrepreneurship with a deep personal involvement, and in situations of personal or work criticality. Thus, they undertake both out of necessity and opportunity, and incorporate a social look of special attention to the other. They develop a characteristic necessity for achievement, associated with overcoming obstacles and reaching proposed goals, although there is also a necessity for affiliation, which is related to the social outlook mentioned above. Finally, the study found a conjunction of collectivist values associated with benevolence, as well as mixed values associated with universalism. The results and their discussion, open up a range of questions and, in turn, of possibilities. The analysis allows us to conclude that questioning the space of women in the entrepreneurial environment and their need for visibility is a must.
\end{abstract}

Keywords: entrepreneurship; female entrepreneurship; female entrepreneurs; entrepreneurial activity; entrepreneurship and gender; entrepreneurship in Argentina; sociodemographic study.

\section{O perfil psicossocial da mulher empreendedora. Uma análise qualitativa e local}

\section{Resumo}

Diante da perspectiva da motivação inicial, da teoria clássica das necessidades e do modelo de valores individualistas e coletivistas, analisa-se o perfil psicossocial da mulher empreendedora da cidade Mar del Plata, na Argentina. Com base etnometodológica, foram aplicados questionários sociodemográficos e entrevistas em profundidade por amostragem orientada, com $\mathrm{N}=34$. A análise de conteúdo das respostas foi realizada de acordo com os modelos teóricos mencionados. As recorrências mostram que as mulheres desenvolvem o seu empreendedorismo com um marcante envolvimento pessoal e diante de situações de críticas pessoais ou profissionais. Assim, comprometem-se tanto por necessidade como por oportunidade e incorporam uma visão social de atenção especial para o outro. Desenvolvem uma característica necessidade de realização, associada à superação de obstáculos e ao alcance das metas propostas, embora exista também a necessidade de filiação, que está relacionada à visão social mencionada. Por fim, foi encontrada uma conjunção de valores coletivistas associados à benevolência, bem como mistos, associados ao universalismo. Os resultados encontrados, assim como sua discussão, abrem um leque de questionamentos e, por sua vez, possibilidades. A análise permite concluir que é necessário questionar o espaço da mulher no campo empresarial e a sua necessidade de visibilidade.

Palavras-chave: empreendedorismo; empreendedorismo feminino; mulheres empreendedoras; atividade empreendedora; empreendedorismo e gênero; empreendedorismo na Argentina; estudo sociodemográfico. 


\section{Profil psychosocial de la femme entrepreneure. Analyse qualitative et locale}

\section{Résumé}

Dans cet article nous analyserons le profil psychosocial des femmes entrepreneures de Mar del Plata, en Argentine, au travers du prisme de la motivation initiale, de la théorie classique des besoins et du modèle de valeurs individuelles et collectives, Les questionnaires sociodémographiques et les entretiens approfondis ont été réalisés via un échantillonnage intentionnel de base ethnométhodologique. L’analyse du contenu des réponses a été réalisée conformément aux modèles théoriques cités précédemment. Les récurrences montrent que les femmes entrepreneures développent leur esprit d'entreprise au travers d'une implication personnelle marquée et lors de situations personnelles ou professionnelles critiques. Elles entreprennent par nécessité et opportunité et sont particulièrement attentives à l'humain. Elles développent une nécessité caractéristique d'accomplissement, franchissant les obstacles et réalisant les objectifs fixés et ont un besoin ou sentiment d'affiliation lié à la perspective sociale mentionnée ci-dessus. Enfin, une combinaison de valeurs collectives associées à la bienveillance et à l'universalisme ont été répertoriées lors de cette recherche. Larticle conclut qu'il est nécessaire de prendre davantage en compte l'espace dédié aux femmes entrepreneures et à leur besoin de visibilité.

Mots-clés: entrepreneuriat; entrepreneuriat féminin; femmes entrepreneures; activité entrepreneuriale; entrepreneuriat et genre; entrepreneuriat en Argentine; étude sociodémographique. 


\section{Introducción}

$\mathrm{E}$

1 desarrollo económico internacional ha llevado, entre otras cuestiones, a una transformación del lugar de la mujer en la sociedad. Esto ha sido denominado por Kanter (1977) como la revolución silenciosa del siglo XX. Pese a estas transformaciones, en la actualidad es aún tema de debate público las brechas de género en el ámbito laboral, lo que atenta contra las posibilidades de igualdad social, económica y política. Es por este motivo que resulta de vital importancia potenciar a la mujer y su contribución a las actividades productivas. Bajo esta premisa el análisis del emprendimiento femenino adquiere gran importancia.

Aunque la actividad emprendedora ha sido analizada por largo tiempo, en la actualidad se reconoce no solo la posibilidad de crear una empresa, sino, principalmente, sus consecuencias sociales: el desarrollo y la dinamización de economías regionales, la creación de empleo, la posibilidad de bienestar social, la posibilidad de un desarrollo laboral equilibrado entre hombres y mujeres. Todos estos motivos han demostrado ser de gran relevancia en la agenda pública internacional, aun con un desarrollo investigativo que no logra acompañar su vertiginoso crecimiento.

Este articulo trata de profundizar esa línea, con base en un estudio empírico realizado a una muestra de mujeres emprendedoras marplatenses. A través de un recorrido por las principales bases de datos - Scopus, Scielo, Latindex - se ha detectado una importante área de vacancia sobre la población aquí analizada. Así, entonces, bajo referentes teóricos tales como la perspectiva de la motivación inicial (GEM, 2016), la teoría clásica de las necesidades (McClelland, 1961) y el modelo de valores individualistas y colectivistas (Schwartz, 1996), este trabajo se plantea el objetivo de conocer el perfil psicosocial de las mujeres emprendedoras de Mar de Plata, Argentina.

Las recurrencias muestran que las mujeres emprendedoras marplantenses desarrollan su emprendimiento con una marcada implicación personal y ante situaciones de criticidad personales o laborales. De esta manera, emprenden tanto por necesidad como por oportunidad e incorporan así una mirada social de especial atención a un otro. También desarrollan una marcada necesidad de logro asociada a la superación de obstáculos y el alcance de metas propuestas, aunque también se encuentra necesidad de afiliación, la cual se relaciona con la mirada social nombrada. Por último, es posible decir que elaboran una conjunción de valores colectivistas asociados a la benevolencia, así como mixtos asociados al universalismo. Tal como se abordará, se cree que el presente trabajo deja pistas para la concreción de medidas que faciliten el camino emprendedor de las mujeres.

La investigación realizada se estructura con esta introducción, seguida por la revisión teórica, en la cual se repasan los conceptos centrales sobre los cuales se construye, es decir, la noción de emprendimiento, de género y emprendimiento, la teoría clásica de las necesidades, el modelo de valores individualistas y colectivistas y la perspectiva de la motivación inicial. A continuación, en un tercer apartado se discuten los resultados propiamente dichos, con una diferenciación entre aquellos cuantitativos y cualitativos. Finalmente, se encuentra la discusión de resultados y la conclusión, en la que se presenta una mirada prospectiva de la temática y se delinean limitaciones de la presente investigación, así como de futuras. 


\section{Revisión teórica}

\subsection{Emprendimiento}

Desde los escritos de Schumpeter (1935) los investigadores han puesto el foco en el análisis de la actividad emprendedora como actividad responsable de los quiebres y progresos del sistema económico, y al emprendedor como el responsable del éxito de la empresa. Sin ahondar en sus diferencias, autores como Kirzner (1973), Knight (1973), Drucker (2002) y Henrekson (2007) han analizado bajo esta lupa al emprendimiento centrándose en su objetivo ulterior: la creación de mayores márgenes de ganancia.

De acuerdo con García y Cañizares (2010), es posible decir que mientras las investigaciones de enfoque mercantilista responden a un análisis del qué, otra gran línea se ha creado dedicada a estudiar el quién, en cuanto análisis del emprendedor en cuestión. Esta línea incluye componentes de la psicología y la sociología, por lo que será denominado como un enfoque psicosocial (Rompato, Silva y Zucarelli, 2018). Esta línea tiene entre sus objetivos conocer los factores sociopersonales, cognitivos, políticos y culturales que determinan la creación y el sostenimiento exitoso de un emprendimiento (Duran y Arias, 2015; Dutra y Rossi, 2011; Narváez, 2012). Además, esta línea ha desarrollado avances sobre lo que se denomina emprendimiento social, entendido como la actividad en la que se elaboran, ejecutan y sustentan iniciativas orientadas a la superación de una dificultad social y el logro de un beneficio común a un grupo humano, bien sea por medio de actividades empresariales o bien socialcomunitarias (Bargsted, 2013).

Para que un emprendimiento sea exitoso es necesario una sólida estructura administrativa colaborativa, coordinada y capaz de generar certidumbre. Así mismo, resulta necesario innovar, ser competitivo y la dirección del proyecto sostenido por un líder que identifique sinergias y alianzas estratégicas, de modo que sean estas últimas entre organizaciones gubernamentales y no gubernamentales, lucrativas y no lucrativas (Fischel, 2013; Silva, 2007; Soto, Acevedo y Labrador, 2015).

La bibliografía también señala que para un correcto desempeño del emprendedor es importante que este presente características de la personalidad que se manifiestan en una forma de pensar y actuar vinculadas a la búsqueda y el aprovechamiento de las oportunidades (Ornelas, Contreras, Silva y Liquidano, 2015). Entre estas se destacan ser optimista, proactivo, persistente y creativo (Durán, 2013). Por su parte, según Bandura (2001), la autoeficacia, entendida como la creencia del individuo sobre su capacidad para realizar actividades con éxito, es importante en el propósito de emprender. De igual forma, investigaciones afirman que el liderazgo, el compromiso y la asunción de riesgos son clave en la creación y el sostenimiento de un emprendimiento (Martínez y Carmona, 2009; Molero, Recio y Cuadrado, 2010; Ortiz, Moncayo y Riaño, 2010; Pino y Aguilar, 2013; Villa y Poblete, 2008). Por último, López, Vázquez y Montes (2010) creen que la resiliencia y las estrategias de afrontamiento son significativos como competencias emprendedoras.

En cuanto a los factores sociodemográficos, Loli, Dextre, Del Carpio y La Jara (2010) encuentran una relación significativa entre la experiencia laboral y el deseo de iniciar un emprendimiento. Bolaños (2006) afirma que, en Latinoamérica, la familia juega un rol importante, así como quienes tienen un rol a imitar, familiar o externo, pueden tener mayor intención de crear un emprendimiento. Por otro lado, en relación con el género, según García y Cañizares (2010), Merino y Vargas (2011), Salazar, Herrera, Rueda y León (2014), las mujeres son menos propensas a iniciar nuevos proyectos empresariales, fruto de su miedo al fracaso. Para otros autores, la propensión hacia la actividad emprendedora está mediada por el nivel de formación/ preparación académica y por la edad (Merino y Vargas, 2011; Rodríguez y Prieto, 2009). 
Independientemente de los diversos resultados, resulta claro que el emprendimiento es una actividad con diversos componentes, más allá del económico.

\subsection{Mujeres emprendedoras}

Desde los comienzos de la legislación laboral la mujer fue excluida del ámbito laboral y recluida en el espacio doméstico. Así se desarrolló un modelo en el que esta era la exclusiva responsable de las actividades domésticas, del cuidado y la educación de los hijos, mientras que el hombre era el responsable de proveer los medios de subsistencia y representar a la familia en el espacio público (Caamaño, 2010). De acuerdo con Siles y Solano (2007), esta división de roles se creó sobre la asunción de que la mujer asume el cuidado como compromiso moral, natural y marcado por el afecto, aunque, lejos de esto, es la normalidad del patriarcado la que ha impuesto dichas diferencias, asignándole un rol social a la mujer cercano a la idea de benevolencia, amor, cuidado, paciencia, etc. Más allá de que el rol cuidador de la mujer sigue activo en nuestra sociedad, en la actualidad los datos demuestran que su ingreso en la actividad laboral es un hecho irrefutable (Tobío, 2012). Aun así, cuando se trata del ámbito emprendedor, es posible anticipar dos cuestiones.

La primera es que cuando se trata de emprender la mujer se encuentra en un claro lugar de inequidad con respecto al hombre. Si bien es posible evidenciar que un número cada vez mayor de mujeres integran actividades productivas y desarrollan sus propios emprendimientos, existen marcadas diferencias de género en el ámbito. A pesar de que socialmente se valore de manera positiva el hecho de que las mujeres emprendan un negocio, no existe igualdad de oportunidades e incentivos con respecto al género masculino, lo que evidencia también un mayor porcentaje de empresas o emprendimientos creados por hombres (García, Ordóñez y Avilés, 2016; Pizarro y Amorós 2006; Saavedra y Camarena, 2015). La bibliografía existente relacionada con el emprendimiento femenino demuestra las dificultades y los obstáculos que deben atravesar las mujeres al iniciar su actividad emprendedora, principalmente, en relación con su género y los estereotipos que, aún hoy en día, se encuentran presentes en el mercado laboral (Cataño y Tamanini, 2015; Heller, 2010; Saavedra y Camarena, 2015). Por otra parte, Karimi, Biemans, Lans, Chizari y Mulder (2014), así como Da Fonseca, Moriano, Laguía y Salazar (2015), han asociado la escasa participación de las mujeres en el ámbito emprendedor a los diversos factores relacionados con la norma subjetiva, entre ellos los condicionamientos sociales y los papeles atribuidos a las mujeres. Esto genera una aprehensión en el momento de emprender, en tanto que la creación de una empresa se percibe como menos favorable por las mujeres (Ramos, Barbera y Sarrió, 2003).

Un obstáculo altamente señalado es el hecho de que la mujer deba conciliar la vida familiar con la laboral (Traverso, Irribarren y Román, 2014; Saavedra y Camarena, 2015). Aunque, a manera de contracara, otras investigaciones ponen de manifiesto la visión que las mujeres tienen del emprendimiento como una posibilidad de generar ingresos y compatibilizar el mundo laboral y familiar (Cataño y Tamanini, 2015; Heller, 2010; Ormeño, 201).

Por último, se ha encontrado que la propia sensibilidad de esta población hacia cuestiones no monetarias suele tener incidencia negativa en el momento de emprender, en la medida en que la decisión de emprender deja de ser solo en términos económicos o de deseabilidad para conjugarse con diversos factores del orden de lo psicosocial (Díaz, Hernández y Barata, 2007; López, 2013; Veciana, Aponte y Urbano, 2005).

La segunda cuestión a destacar es que el emprendimiento adquiere matices propios cuando se trata de mujeres. Un análisis realizado por Guerrero, Canibe, Armenteros, López y Reyna (2012) en México describe a la mujer emprendedora con una edad promedio de 47 años, y un $68 \%$ con educación universitaria. En un $47 \%$ han emprendido por una conjunción de motivos, mientras que un $27 \%$ lo ha hecho única y exclusivamente por necesidad. El $41 \%$ ha emprendido en el rubro de servicios, principalmente, en los sectores de salud, educación, 
alimentos, bebidas y hospedaje, y el $77 \%$ tiene a su cargo dependientes económicos, bien sean estos hijos o bien familiares. En cuanto a su perfil por competencias, las emprendedoras mexicanas han sido descriptas como comprometidas, participativas, intuitivas, creativas, adaptables, independientes, trabajadoras y seguras de sí mismas.

Sin embargo, la realidad latinoamericana es altamente heterogénea, de modo que es posible encontrar diferencias entre las investigaciones. Si volvemos sobre las palabras de López et al. (2012), encontraremos que existen factores externos que condicionan tanto al emprendimiento como al emprendedor, los cuales son el ámbito en el que se desempeña y el entorno económico regional. Por tanto, aunque el trabajo referenciado se ha realizado en Latinoamérica, es importante destacar que Rábago, D'Annunzio y Monserrat (2004) han descrito el espíritu emprendedor de la mujer argentina de distinta manera. Según estas investigadoras, este posee:

- Elevado nivel de autovaloración de sus competencias personales y empresariales. Esto implica responsabilidad individual, habilidades organizativas y un importante grado de autonomía e independencia.

- Presencia de foco de control interno. Este se refleja, principalmente, en el manejo adecuado de la información, una alta necesidad de logro, representada en características tales como capacidad de organización, identificación de metas claras, vigor y determinación, así como alta capacidad de perseverancia.

- Afiliación a mentores vinculados a la carrera empresarial y al aspecto psicosocial. Indica que las emprendedoras tienden a «imitar» modelos de comportamiento diseñados y desarrollados por los individuos con quien ellas se identifican.

- Aplicación del concepto de «aprendizaje delegado» a sus propios aprendices.

- Un estilo de liderazgo abierto y participativo.
Hisrich (2008), García y Espino (2013), así como López et al. (2012), afirman que las mujeres se encuentran motivadas por el deseo de ser independientes y autosuficientes, por el descontento con la situación laboral/profesional y económica actual, así como por la posibilidad de tomar decisiones. De igual forma, describen a la mujer emprendedora como flexible $y$ tolerante, orientada a metas, creativa, realista y con experiencia en su rubro de trabajo. Por último, reconocen cómo la mujer que incursiona en la actividad emprendedora lo hace aun sabiendo que dicha incursión pudiese tomar más tiempo que a un hombre.

Por otra parte, estudios previos confirman que las mujeres confían menos que los hombres en sus habilidades para emprender (Langowitz y Minniti, 2007; Oliveira, Moriano, Laguía y Salazar, 2015; Wilson, Kickul y Marlino, 2007). Según Da Fonseca et al. (2015), este resultado no permite afirmar que los hombres atribuyan mayor importancia a valores individualistas que las mujeres, aunque sí mantienen distintos patrones en cuanto a estos. En relación con los valores colectivistas, estos autores han encontrado que la conformidad y benevolencia influyen de forma negativa sobre la intención emprendedora de las mujeres.

Ormeño (2014) también encuentra que las mujeres están motivadas por valores colectivistas, aunque de manera positiva, en la medida en que se enfocan en generar trabajo a otros y traspasar lo aprendido. Esta autora afirma, además, que la mujer emprendedora no presenta una alta motivación al logro, aunque sí una fuerte necesidad de afiliación. También explica que para las mujeres el emprendimiento es una forma de generar ingresos, pero sin descuidar a la familia, y que, más aun, sirve como un espacio de desarrollo personal, autoafirmación y recuperación de la noción de género.

Cedeño (2013), por su parte, señala que para las mujeres el emprendimiento es una forma de compatibilizar el ejercicio profesional y la vida familiar, y cómo, pese a las diferencias de cada emprendimiento, el problema en común con el que se encuentran las mujeres es el desconocimiento del área administrativa. 


\subsection{Teoría de las necesidades}

Con una vasta bibliografía sobre la temática (Alderfer, 1972; Maslow, 1954; McClelland, 1961) se ha optado por analizar a las mujeres emprendedoras con el marco teórico propuesto por McClelland (1961), quien plantea la existencia de tres necesidades: logro, poder y afiliación. Los individuos establecerán metas, objetivos, comportamientos y caminos distintos, dependiendo de cuál de estas tres necesidades predomine. Estas se definen de la siguiente manera:

- Necesidad de logro. Es la tendencia a vencer obstáculos, realizar nuevos retos y cumplimentar logros. La persona con necesidad de logro asume la responsabilidad por su éxito o fracaso y prefiere correr riesgos moderados que entreguen una retroalimentación instantánea sobre su actividad. Fruto de su responsabilidad, suele tener problemas para delegar autoridad. No se encuentra tan estimulado por la recompensa monetaria, sino por la satisfacción de logro y realización.

- Necesidad de afiliación. Refiere a la necesidad de afecto e interacción con los demás. Las personas con alta necesidad de afiliación encuentran satisfacción en actividades sociales $\mathrm{e}$ interpersonales que requieran de fuertes lazos interpersonales. Así mismo, se caracterizan por mostrar una gran preocupación con respecto al reconocimiento ajeno y a preferir trabajar en grupo.

- Necesidad de poder: Hace referencia a la necesidad de influir, dirigir y controlar a otras personas. Las personas con tendencia a la necesidad de poder no encuentran tanta satisfacción en la actividad en cuestión, sino en mantener una posición que detente influencia o control.

\subsection{Motivación inicial}

Otro de los ejes a indagar fue la motivación inicial a emprender, diferenciada a través de los conceptos de necesidad u oportunidad. Estos conceptos los analiza el Global Entrepreneurship Monitor (GEM, 2016) a través del Índice Motivacional, el cual mide el porcentaje de personas involucradas en emprendimientos nacientes que están motivadas por oportunidades de mejora, divididas por el porcentaje de emprendimientos nacientes motivados por necesidad. En el caso específico de las mujeres, el GEM mide la proporción de mujeres participantes en la actividad emprendedora motivadas por la oportunidad. Esta proporción responde al porcentaje de mujeres involucradas en emprendimientos nacientes que 1) dicen ser impulsadas por la oportunidad en lugar de no encontrar otra opción para el trabajo; y 2) quienes indican que el principal impulsor para participar en esta oportunidad es ser independientes o aumentar sus ingresos, en lugar de simplemente mantener sus ingresos, dividido por el porcentaje equivalente para sus homólogos masculinos.

Profundizar en el concepto de oportunidad permite realizar un análisis aún más interesante del rol de la mujer en el emprendimiento, dado que nos permite conocer cuánto de la participación en dicha actividad está relacionada con los nuevos roles que las mujeres, de forma paulatina, comienzan a tener en la sociedad, o si simplemente responde a una mera necesidad económica.

\subsection{Modelo de valores}

Por último, se analizaron los valores, los cuales son básicos en nuestro accionar como seres humanos. Estos permiten a nivel social compartir normas e identificar el comportamiento deseado y esperado en diversas situaciones. Así también, a nivel individual funcionan como metas deseables y transituacionales que varían de importancia y sirven como principioguía en la vida de las personas (Schwartz, 1996). Schwartz (1996) propone una estructura formada por diez tipos de valores que se agrupan en tres dimensiones: individualismo, colectivismo y valores mixtos. El individualismo puede encontrarse en valores como, por ejemplo, poder, logro, hedonismo, estimulación y autodirección. El colectivismo se traduce en valores tales como benevolencia, tradición y conformidad. Por último, se encuentran valores mixtos en valores como el universalismo y la seguridad. A modo de ánalisis, 
cada uno de estos tipos de valor se traduce a valores específicos tales como autoridad, creatividad, perdón, obediencia y justicia social, entre otros. En el caso de las mujeres entrevistadas, se ha encontrado que están altamente motivadas por valores colectivistas $y$, en cierta medida, por valores mixtos.

\section{Metodología}

Se trata de una investigación prospectiva, Sexploratoria descriptiva, de corte transversal, con un enfoque cuanti y cualitativo, aunque con una fuerte incidencia de esta última. La elección de esta metodología responde al propio objetivo del enfoque cualitativo, la orientación hacia la acción humana y la subjetividad, de modo que se destaca al sujeto como núcleo central del estudio, en la medida en que es el propio entendimiento que los sujetos hacen de sus vidas y el mundo que los rodea (Mejía, 2000).

De igual forma, la corriente etnometodológica propuesta tiene como base el reconocimiento de las actividades fundamentales que componen el emprendimiento y dotan de sentido a esta actividad en cuestión. En términos de Garfinkel (1991), se trata de reconocer el sentido y la racionalidad práctica que se enlaza a dicha actividad.

A partir de la realización de talleres sociales en la Universidad Nacional de Mar del Plata (UNMDP), orientados a desarrollar competencias psicosociales emprendedoras $^{3}$, las mujeres participantes fueron invitadas a hacer parte de la presente investigación, de manera que resultó una muestra intencional de 34 mujeres emprendedoras.

La investigación constó de dos momentos. El primero fue de desarrollo cuantitativo, en el cual se administró un cuestionario sociodemográfico desarrollado por el Grupo de Investigación en Psicología Laboral (GIPsiL), de la Facultad de Psicología de la UNMDP. Este se concentró en la recolección de datos referidos a edad, lugar de nacimiento, características socioeducativas, estado civil, cantidad de hijos y personas a cargo. Luego, se realizaron entrevistas en profundidad realizadas a las mujeres emprendedoras. Las entrevistas tuvieron como objetivo conocer aquello que constituye a la mujer emprendedora desde su propia voz: rubro de pertenencia del emprendimiento, motivaciones, necesidades y valores, entre otros.

Una vez se recogieron los datos, se procedió a cargarlos en sus respectivas matrices. En el caso de aquellos obtenidos mediante metodología cuantitativa se analizaron por medio del software SPSS, para lo cual se crearon descriptivos básicos y tablas de contingencia. La información recabada mediante instrumentos cualitativos se analizó con el programa Atlas.ti, con el foco en la creación de códigos y redes. A partir de estos se buscó no solo interpretar la información, sino también generar nodos que demostrasen la pertinencia y relación entre los conceptos analizados. Para finalizar, se realizó análisis de contenido de las respuestas de acuerdo con los modelos teóricos mencionados. Estos datos permitirán contextualizar a la mujer emprendedora marplatense, de la que hasta hoy no se han obtenido información.

\footnotetext{
${ }^{3}$ Específicamente, se trata de un proyecto de extensión denominado Mujeres Emprendedoras, dependiente de la Facultad de Psicología de la mencionada universidad.
} 
Tabla 1. Resumen de operacionalización de variables incluidas en la investigación

\begin{tabular}{|c|c|c|c|}
\hline Enfoque & Variable & Dimensión de la variable & Nivel de medición \\
\hline \multirow{6}{*}{ Cuantitativo } & Edad & Pregunta abierta & Ordinal \\
\hline & Lugar de nacimiento & $\begin{array}{l}\text { Mar del Plata } \\
\text { Otros }\end{array}$ & Nominal \\
\hline & Estado civil & $\begin{array}{l}\text { Soltera } \\
\text { Casada } \\
\text { Divorciada } \\
\text { Viuda }\end{array}$ & Nominal \\
\hline & Características socioeducativas & $\begin{array}{l}\text { Primario incompleto } \\
\text { Primario completo } \\
\text { Secundario incompleto } \\
\text { Secundario completo } \\
\text { Terciario incompleto } \\
\text { Terciario completo } \\
\text { Universitario incompleto } \\
\text { Universitario completo }\end{array}$ & Ordinal \\
\hline & Cantidad de hijos & Pregunta abierta & Ordinal \\
\hline & Personas a cargo & $\begin{array}{l}\text { Sí } \\
\text { No }\end{array}$ & Nominal \\
\hline \multirow{4}{*}{ Cualitativo } & Rubro del emprendimiento & Pregunta abierta & No corresponde \\
\hline & Motivaciones & $\begin{array}{l}\text { Necesidad } \\
\text { Oportunidad }\end{array}$ & No corresponde \\
\hline & Necesidades & $\begin{array}{l}\text { Logro } \\
\text { Afiliación } \\
\text { Poder }\end{array}$ & No corresponde \\
\hline & Valores & $\begin{array}{l}\text { Colectivista } \\
\text { Individualista }\end{array}$ & No corresponde \\
\hline
\end{tabular}

Fuente. Elaboración propia. 


\section{Resultados}

\subsection{Variables sociodemográficas}

De la muestra analizada, se puede señalar que las mujeres emprendedoras marplatenses tienen, en promedio, 39 años; el $70 \%$ de ellas nació en la provincia de Buenos Aires, con fuerte incidencia de la ciudad de Mar del Plata, seguido del $14 \%$ en la Ciudad Autónoma de Buenos Aires (CABA). El $41 \%$ presenta estudios terciarios completos $^{4} \mathrm{y}$ el $26 \%$ ha realizado hasta el secundario. En tercer lugar, pero aún importante, se encuentra que el $23 \%$ ha realizado y completado estudios universitarios. En cuanto a su estado civil es posible señalar que la amplia mayoría de las mujeres entrevistadas es soltera, específicamente el $41 \%$ de la muestra, mientras que el $32 \%$ es casada; el porcentaje restante, el $26 \%$, se encuentra divorciada. Es importante destacar que no se han encontrado mujeres en situación de viudez. En lo que refiere a las personas a cargo de las emprendedoras, entendiéndolas como hijos o terceros, se han encontrado porcentajes dispares. El 33 \% tiene un solo hijo a cargo, mientras que el $23 \%$ de las mujeres no tiene hijos a su cargo y, con el mismo porcentaje, el $23 \%$, corresponde a mujeres que tienen tres o más hijos. Como tercera medida encontramos a aquellas mujeres que tienen dos hijos a cargo, con un $20 \%$. Como se verá más adelante, los hijos constituyen un factor crucial en el camino emprendedor de las mujeres. Por último, en lo que respecta a «otras personas a cargo», se ha encontrado que el $94 \%$ declara no tenerlas, en contrapartida con el $6 \%$ que sí, y son estos, en general, padres o madres mayores.
En resumen, y de acuerdo con los 34 casos analizados, los resultados muestran que la mujer emprendedora marplatense es una mujer en edad adulta, con estudios intermedios cursados, aunque también se ha encontrado un porcentaje considerable de mujeres con estudios de grado. Esto deja entrever que su relación con el emprendimiento, en mayor o menor medida, no es improvisada, sino que se asienta en conocimientos. Por otra parte, en su amplia mayoría, las mujeres emprendedoras no tienen pareja, al menos desde la legalidad. Esto se da tanto porque es soltera o porque está divorciada. Tampoco suelen tener terceros a cargo por fuera de sus hijos.

\subsection{Análisis cualitativo}

El análisis cualitativo está compuesto de una batería de preguntas que intentan dar una descripción acabada de la mujer emprendedora; es por esto que estas hacen un recorrido desde lo general a lo particular. Bajo esta lógica resulta importante conocer, primero, el tipo de emprendimientos al que las mujeres suelen dedicarse, dado que dará una clara contextualización de la información posterior.

\subsubsection{Rubro de pertenencia del emprendimiento.}

Las mujeres declaran tener emprendimientos dedicados a la producción de bienes materiales o enfocados en la prestación de servicios. La muestra se encuentra dividida en partes casi iguales entre ambos tipos de emprendimientos, con una leve mayoría de mujeres dedicadas a la producción de bienes materiales.

\footnotetext{
${ }^{4}$ En Argentina, la educación superior está comprendida por la educación universitaria y terciaria. Esta última se desarrolla en institutos de educación superior o universidades, tiene una duración de entre seis meses y tres años, y está orientada a títulos «técnicos», no profesionales.
} 
Resulta interesante destacar que la producción de bienes materiales se encuentra estrechamente ligada a un trabajo que remite a lo artesanal, a lo natural, a la creatividad y a la creación con las propias manos. De hecho, los emprendimientos descritos responden a trabajos socialmente aceptados como femeninos y poco asociados a un nivel alto de profesionalización. Es así como se han encontrado emprendimientos dedicados a la producción de comida vegana, al reciclado de muebles, indumentaria con imprenta casera, portabebés ergonómicos y remeras para amamantar, carteras con cuero ecológico o conservas alimenticias artesanales, entre otros. Para las mujeres la producción supone poner su propio cuerpo y mente, imprimiendo un sello único a su producto. Esto genera dos grandes consecuencias: originalidad en el producto desarrollado y, como contraparte, la imposibilidad de una creación a gran escala.

En lo que respecta a los servicios se encuentra la misma tendencia: mujeres que ponen su creatividad y desarrollo personal al servicio de terceros. Aun con más incidencia que en la producción de bienes materiales, se encuentra que el desarrollo de servicios suele estar ligado a experiencias personales sumadas a capacitaciones en el área. Así, mientras que se ha destacado la producción de comida vegana, en el área de servicios es también posible encontrar desarrollos gastronómicos. La diferencia radica en toda la experiencia de compra-venta que la emprendedora logra generar gracias a la capacitación que posee y lleva, por ejemplo, a un servicio de catering. Esta diferenciación se explica, principalmente, porque aquellas mujeres que emprenden dentro del rubro de los servicios suelen tener un mayor nivel de formación con relación a aquellas que lo hacen dentro de la producción de bienes materiales. Otro ejemplo de este mayor nivel de formación remite al desarrollo de mujeres emprendedoras fotógrafas, grafólogas, profesoras o bioquímicas que se ha encontrado en la muestra entrevistada.

Pareciera que en el caso del área de servicios la mujer emprendedora se formó $y$, de manera consciente, ejercitó una conducta emprendedora al pensar, planificar y organizar su trabajo. Mientras que, en el caso de las mujeres dedicadas a la producción, en muchas ocasiones se «han encontrado» vendiendo aquello que producían para su hogar o amistades, lo que supone un estado de desconocimiento de la actividad más marcado.

Aun así, es interesante destacar que tanto en el área de producción como de servicios abunda la implicación personal. En ambos casos las mujeres se proyectan como emprendedoras comprometidas con su labor, con su desarrollo y sus clientes. De hecho, aunque muchas de ellas se han encontrado emprendiendo sin buscarlo de manera activa, la actividad seleccionada para tal fin responde a una necesidad personal e íntima que ha sabido traducirse en un comercio, lo cual explica la marcada implicación personal encontrada.

Una mención aparte merece la connotación social de los emprendimientos, sean estos de producción de bienes o servicios. De manera grata se ha encontrado en las mujeres emprendedoras que por propia necesidad o curiosidad han sumado componentes a su emprendimiento que lo configuran como uno de índole social. Este tipo de emprendimiento se caracteriza por buscar oportunidades que generen no solo valor económico, sino valor social, a partir de la solución de problemáticas sociales de forma efectiva, eficiente y sustentable (Palacios, 2010; Roberts y Woods, 2005). Si bien no existe una definición generalizada, se concuerda en que el emprendimiento social, incluso al compartir características comunes con el emprendimiento convencional, tiene como finalidad o propósito llevar a la resolución de problemas sociales (Bargsted, 2013; Guzmán y Trujillo, 2008; Jirón y Chan, 2016).

En el caso de las mujeres emprendedoras se ha encontrado que estas han ido adoptando patrones propios del tipo de emprendimiento descrito. Este es el caso de la emprendedora dedicada a producir y comercializar juegos inclusivos para niños con discapacidades psicomotrices, así como el de aquella que produce cosméticos de origen natural, sin conservantes ni pesticidas. Estos casos responden a emprendimientos cuya finalidad es económica, pero sin olvidar el componente social. Sin embargo, 
también se ha encontrado el caso de una mujer que ha emprendido una cooperativa dedicada al saneamiento ambiental. Este emprendimiento no persigue un lucro económico, sino únicamente un fin social. Es importante destacar este caso, dado que, históricamente, el emprendimiento ha sido asociado a una finalidad económica, de manera que las actividades no lucrativas se consideraban simplemente ociosas. El concepto de emprendimiento social logra dotar de pertinencia a este tipo de emprendimientos.

Aunque en términos cuantitativos resulta pequeño el porcentaje de mujeres que suma componentes sociales a su emprendimiento, es interesante ver cómo este fenómeno, de reciente clarificación teórica, comienza a desarrollarse en la práctica.

\subsubsection{Motivación.}

Aun cuando la producción de datos comenzó recién en el 2013, resulta alentador ver que en Argentina una amplia mayoría de las mujeres emprendedoras lo son por la oportunidad que esto significa, no por necesidad. El menor valor encontrado corresponde al periodo 2013-2016, y fue de 0,79 mujeres emprendedoras por cada hombre emprendedor, mientras que en el 2017 se registró 0,94 mujeres emprendedoras por oportunidad de cada hombre emprendedor. Efectivamente, bajo un contexto que propicie la situación, la mujer deja de lado su rol más tradicional para conocer sus oportunidades en el mundo laboral de la mano del emprendimientoo (Rompato, 2018).

Pese a la información brindada por el GEM es importante destacar que, aunque a nivel teórico se realice esta diferenciación, en la realidad las motivaciones suelen ser múltiples, en tanto los límites entre una situación personal y las consecuencias de la misma suelen ser difusos. Por este motivo resultó necesario indagar de forma cualitativa a las mujeres emprendedoras, a fin de conocer no solo su motivación inicial, sino también dichas situaciones personales que pudiesen llevar a la acción de emprender.
Las entrevistas han dejado entrever que, en cantidades similares, las mujeres emprenden tanto por necesidad como por oportunidad. En el caso de la motivación por necesidad se encuentran dos patrones que se repiten: mujeres que emprenden después de un divorcio y aquellas que emprenden manteniendo como norte a sus hijos.

Bajo el primer patrón se ha encontrado que, fruto de situaciones de violencia de género, las mujeres deciden divorciarse. Sin embargo, al igual que la relación en cuestión, el divorcio suele ser traumático, dado que las mujeres se encuentran despojadas no solo de bienes materiales, sino de su propia identidad. La conjunción de ambas cosas las lleva a reencontrarse con viejos hobbies o conocimientos que, eventualmente, se traducen en la creación de un emprendimiento. Bajo esta lógica no sorprende la implicación personal antes comentada, en la medida en que el emprendimiento deja de ser solo por una necesidad económica para ser un «salvavidas» ante tal situación de criticidad.

En el caso del segundo patrón encontrado, aquellas mujeres que emprenden por necesidad y consideran como norte a sus hijos, se han encontrado dos situaciones: quienes luego de la maternidad han decidido dejar su trabajo en relación de dependencia, pero, como de todas formas necesitan trabajar, han decidido emprender y realizar sus actividades desde el hogar. Esto las lleva a cumplir y, en muchos casos, superponer las actividades familiares y laborales. Por otra parte, se encuentran las mujeres que han decidido emprender para dejar una suerte de legado a sus hijos. En lo que respecta a este último caso se ha detectado, en específico, mujeres cuyos hijos sufren alguna enfermedad o condición, por lo que han decidido emprender para que ellos puedan incorporarse al mercado laboral.

\subsubsection{Necesidades: afiliación, poder, logro.}

$\mathrm{Al}$ igual que con el reconocimiento de la motivación inicial, conocer la necesidad predominante en las mujeres emprendedoras resulta necesario en la medida en que su satisfacción permitirá generar una mayor influencia sobre la motivación. Es decir, se presenta una suerte de círculo virtuoso entre ellas. 
En el caso de las mujeres emprendedoras, se ha encontrado que presentan necesidad de logro y de afiliación, lo cual muestra una leve tendencia hacia la primera. Aunque en primera instancia podría considerarse que la necesidad de afiliación sería la predominante, fruto de los emprendimientos con una marcada visión social, seha encontrado quelas mujeres, ante todo, desean cumplir con su meta propuesta. Sea quelas mujeres emprendan por una situación personal, algo más relacionado a la necesidad, o por el deseo de independencia y crecimiento económico, relacionado con la oportunidad, las mujeres emprendedoras mantienen como norte su necesidad de lograr aquello que se han propuesto, trabajando con determinación y de manera incansable. Es por esto que «hacer lo que les gusta», «vivir de su emprendimiento»o «ser reconocidas en su materia» se presentan como hechos paradigmáticos. Para estas mujeres el logro más grande reside en haber sorteado limitaciones, propias y ajenas, $y$ en «animarse» a emprender. Es por esto que encuentran tanta satisfacción cuando el resultado es positivo.

No obstante, también presentan signos de necesidad de afiliación, pues la propia estructura de los emprendimientos hace que esto resulte obvio. Se ha encontrado que conocer gente, ayudar, el agradecimiento recibido $y$, en especial, cuando el cliente se encuentra a gusto con el producto o servicio ofrecido, resultan claros estímulos satisfactorios.

\subsubsection{Valores individualistas, mixtos y colectivistas.}

Todos las emprendedoras y los emprendimientos hasta ahora descritos responden a una lógica colectivista que busca generar no solo un bienestar económico individual, sino, sobre todo, dar respuestas a necesidades de otros: a los hijos, en general, pero también la sociedad en un sentido amplio. Esta mirada social, relatada a lo largo del presente estudio, remarca el fuerte componente colectivista presente en las mujeres emprendedoras. Bien sea porque, de manera consciente, deciden dar respuesta a una problemática social, como, por ejemplo, la ecológica o la inclusión social, o bien porque deciden que aquello que les permitió soportar situaciones de criticidad sea también conocido por un tercero, las mujeres emprendedoras tienden a generar emprendimientos que no solo sean económicamente redituables, sino que también sirvan como experiencia y aprendizaje para sus clientes.

Este tipo de comportamiento está estrechamente relacionado con la benevolencia, en la medida en que la mujer emprendedora intenta, ante todo, ayudar al otro. También, y de acuerdo con su histórico rol de cuidadora socialmente impuesto (Siles y Solano, 2007), puede pensarse desde la necesidad de mantener dicho rol más allá de las esferas privadas del hogar.

Por otra parte, se encuentra el componente del perdón en cuanto valor específico. Como ya ha sido relatado, el emprendimiento muchas veces se entiende como soporte ante situaciones de criticidad, como una forma de perdonar y reinventarse ante el dolor.

De acuerdo con esta misma lógica de mirada hacia el otro, es importante destacar la presencia de valores mixtos. Las mujeres emprendedoras muestran claros signos de valores mixtos tales como el universalismo, registrados a través de la justicia social y la igualdad que, consciente o inconscientemente, pregonan con sus emprendimientos. 


\section{Discusión de resultados}

A partir delos resultados del estudio es posible decir que las mujeres emprendedoras marplatenses tienen, en promedio, 39 años de edad, y que un $41 \%$ posee estudios terciarios. Así también se ha registrado un $23 \%$ de la muestra que ha realizado y terminado sus estudios universitarios. El $41 \%$ es soltera, el $32 \%$ es casada y un $26 \%$ se encuentra divorciada. Un $76 \%$ tienen hijos a cargo, mientras que solo un $6 \%$ tiene otras personas a cargo, y son estos madres o padres mayores. Si se compara con la investigación realizada por Guerrero et al. (2012), es posible decir que un porcentaje menor de las emprendedoras analizadas poseen estudios universitarios, aunque casi el mismo porcentaje de emprendedoras tanto argentinas como mexicanas tiene a su cargo dependientes económicos.

Los emprendimientos pueden dividirse en sector productivo o de prestación de servicios. En ambos casos se han encontrado actividades socialmente consideradas femeninas, tales como aquellas relacionadas con la cocina, el cuidado del hogar, los niños, etc. Así mismo, para ambos casos se ha percibido una marcada implicación personal, lo cual se encuentra relacionado con una necesidad personal e íntima que ha sabido traducirse en un emprendimiento. Se cree que, en última instancia, ambas cuestiones se relacionan con el rol que, históricamente, ha ocupado la mujer en la sociedad, por el cual, más allá de que atienda una tarea laboral, lo realiza mediante comportamientos típicamente asignados al género femenino, con benevolencia, amor y una gran implicación personal (Caamaño, 2010; Siles y Solano, 2007; Tobío, 2012). Por último, en el caso de los emprendimientos relacionados con la prestación de servicios, se percibe un mayor grado de formación de la mujer.

En cuanto a la motivación inicial, es posible decir que las mujeres emprenden tanto por necesidad como por oportunidad. En el caso de las primeras, se ha encontrado que el desarrollo de emprendimiento surge a partir de situaciones de criticidad, como, por ejemplo, divorcios, y tienen como eje la necesidad de proveer a los hijos. De acuerdo con la bibliografía, la maternidad es tanto un puntapié como una complicación en el momento de emprender (Cataño y Tamanini, 2015; Heller, 2010; Ormeño, 2014; Saavedra y Camarena, 2015; Traverso et al., 2014). En este caso encontramos que, para las mujeres entrevistadas, el emprendimiento es una respuesta a sus necesidades personales y económicas que supone, en gran parte, esa maternidad. Bien sea porque deciden trabajar dentro de su hogar y, de forma simultánea, cuidar de sus hijos, o bien para incorporar a un hijo al mercado laboral, las mujeres compatibilizan su vida familiar y laboral mediante la actividad emprendedora.

Por otra parte, se encuentran mujeres que, motivadas por el hastío generado hacia el trabajo en relación de dependencia y el gusto intrínseco por la independencia, deciden emprender. Esta conjunción las ha llevado tomar la resolución de emprender como respuesta, en busca de actividades que pudieran compatibilizar con dichas necesidades. Es decir, el emprendimiento se presenta como una oportunidad. Esta afirmación se encuentra en consonancia con diversas investigaciones que afirman el deseo de dependencia y autosuficiencia, así como el descontento laboral/profesional que lleva a la mujer a emprender (García y Espino, 2013; Hisrich, 2008; López et al., 2012).

Más allá de lo económico, las mujeres emprendedoras presentan una estrecha relación con elementos psicosociales de un emprendimiento, expresados, principalmente, por medio de un marcado interés por un otro. Este motivo las lleva, muchas veces, a generar emprendimientos de perspectiva social, con base en el desarrollo de actividades que puedan servirle a otro de la misma forma que lo hicieron con ellas. Tal es el caso, por ejemplo, de las emprendedoras que ante la necesidad de trabajar y conciliar la crianza de sus hijos han decidido producir bienes para una crianza 
responsable en espera de que otras familias conocieran sus bondades, o quienes proponen emprendimientos orientados a masificar actividades recreativas que las han ayudado a superar situaciones críticas, tales como el teatro o las biodanzas.

Esta situación encuadra con lo que se denominó emprendimiento social (Bargstad, 2013), mediante el cual las mujeres emprendedoras buscan generar un medio de subsistencia, aun dando respuesta a diversas problemáticas sociales. En otros casos se ha encontrado que se emprende por la oportunidad de hacer algo que genera satisfacción, independientemente de la variable económica. Aunque esta justificación no responde a la definición que comúnmente se establece del emprendimiento o a la idea de oportunidad propuesta por el GEM (2016), contiene el mismo valor para las mujeres entrevistadas, en la medida en que la oportunidad radica en explorar un costado personal o profesional que de otra manera no podrían.

Bien sea que las mujeres emprenden por necesidad o bien por oportunidad, han demostrado mantener una alta necesidad de logro que se traduce en un trabajo determinado e incansable. Así también, conocer gente, ayudar, el agradecimiento recibido $y$, en especial, cuando el cliente se encuentra a gusto con el producto o servicio ofrecido, resultan claros estímulos satisfactorios - esto se relaciona con una necesidad de afiliación-. Parecería posible pensar que la necesidad de logro es aquello que guía a las mujeres emprendedoras, mientras que la necesidad de afiliación es la que acompaña este camino emprendedor.

El logro no está de forma directa relacionado con lo económico, aunque tampoco se niega su importancia; para estas mujeres tiene que ver con superar obstáculos y alcanzar las metas propuestas, ya sea lograr independencia, reencontrarse con su propia identidad, compatibilizar el trabajo con el cuidado de los hijos, etc. Mientras que la necesidad de afiliación es aquello que acompaña, que da aliento cotidiano para sostener sus metas en el largo plazo y genera así la cuota necesaria de perseverancia cuando la necesitan.
Por último, las mujeres emprendedoras presentan valores colectivistas tales como la benevolencia, desarrollada, principalmente, por medio de la necesidad de ayudar y del sentido del perdón que invoca al emprendimiento en sí. De igual forma, se han encontrado valores mixtos tales como el universalismo, registrados a través de la justicia social y la igualdad que, consciente o inconscientemente, pregonan con sus emprendimientos.

$\mathrm{Al}$ establecer una relación entre los valores y la actividad emprendedora, se verifica que el papel de los valores en la comprensión del perfil emprendedor merece más atención de la que actualmente tiene. Aunque se han detectado investigaciones destinadas a conocer, entre otras cuestiones, los valores de las mujeres emprendedoras (Da Fonseca et al., 2015; Ormeño, 2014), en líneas generales el análisis se ha direccionado a conocer sus competencias (López et al.,2012; García y Espino, 2013; Rábago, D 'Annunzio y Monserrat, 2004).

Al mantener una mirada ambigua sobre los valores colectivistas, la bibliografía desarrollada hasta el momento indica también que las mujeres emprendedoras se guían por dichos valores. Aunque el alcance de la presente investigación imposibilita conocer la relación que existe entre los valores encontrados y el éxito futuro de los emprendimientos, es importante rescatar que, aunque sea de momento, son esos mismos valores, desarrollados a través de valores específicos tales como el perdón, la ayuda, la igualdad y la justicia social, los que influyen y organizan el comportamiento emprendedor de las mujeres.

Resulta interesante destacar que la única investigación argentina sobre la temática también destaca la necesidad de logro de las mujeres emprendedoras y encuentra en estas la marcada capacidad de organización, la identificación de metas claras, vigor y determinación, así como alta capacidad de perseverancia; todas características propensas a determinar un fuerte perfil emprendedor (Rábago et al., 2004). 


\section{Conclusiones}

$\mathrm{E}$ ste trabajo se desarrolló a partir de un área de vacancia encontrada -el análisis de la mujer emprendedora marplatense- con el ánimo de colaborar en su fortalecimiento. En este sentido, se desarrolló la investigación centrada en analizar el perfil psicosocial de dichas mujeres mediante el uso de tres teorías: la teoría clásica de las necesidades (McClelland, 1961), el modelo de valores individualistas y colectivistas (Schwartz, 1996) y la perspectiva de la motivación inicial (GEM, 2016).

Los resultados encontrados, así como su discusión, abren un abanico de preguntas y, a su vez, de posibilidades. En primera instancia, es necesario cuestionarse el espacio de la mujer en el ámbito emprendedor y su necesidad de visibilización. Tal como afirma Tobío (2012), a esta altura no cabe discusión sobre la inserción de la mujer en el mercado laboral, ¿pero qué lugar ocupa en el ámbito emprendedor? Para comenzar, es interesante destacar que, si bien aquí se trabajó con mujeres que efectivamente emprenden, diversas investigaciones destacan que el universo emprendedor es socialmente considerado un ámbito masculino, en el cual las mujeres, debido a su supuesta sensibilidad, no tienen espacio de participación (Díaz et al., 2007; López, 2013; Veciana et al., 2005). Sin embargo, para aquellas que deciden participar, la bibliografía analizada muestra que no existe igualdad de oportunidades e incentivos con respecto al género masculino, lo que evidencia un mayor porcentaje de empresas o emprendimientos creados por hombres (García et al., 2016; Pizarro y Amorós, 2006; Saavedra y Camarena, 2015). Así mismo, más allá de que la muestra analizada afirme que el emprendimiento se percibe como una fuente de ingresos económicos para su familia, el impuesto y naturalizado rol de cuidadora obliga al género femenino a organizar su dinámica laboral con base en sus obligaciones familiares, hecho infrecuente en los hombres (Saavedra y Camarena, 2015; Traverso et al., 2014).

Estas situaciones conllevan un sinfín de complicaciones para las mujeres que deciden aventurarse en el mundo emprendedor, por lo cual, tal como se afirma en la introducción, resulta necesario desandarlas, pensarlas y utilizarlas a fin de generar medidas que faciliten su camino. Algunas de las tareas necesarias y posibles para llevar a cabo desde el ámbito educativo son la educación sobre el ámbito emprendedor, con la inclusión de conocimientos administrativos, y el desarrollo de competencias estratégicas tales como liderazgo, resiliencia, trabajo en equipo, etc. Por otra parte, a nivel gubernamental, podrían considerarse ayudas económicas específicas para mujeres emprendedoras, espacios de difusión de sus emprendimientos y políticas estratégicas que fortalezcan a la mujer, de manera que desnaturalicen, por ejemplo, su rol cuidador. El camino es largo, pero el deseo es aún mayor. De este modo, se espera que los resultados encontrados se puedan utilizar no solo como cimiento de futuras investigaciones, sino dirigidas a cualquier persona interesada en la temática emprendedora, en la relación entre género y emprendimiento $y$, principalmente, a agencias gubernamentales interesadas en fortalecer a su comunidad emprendedora.

Para finalizar, es necesario destacar las limitaciones del presente estudio. Desde una lógica cuantitativa es menester afirmar que los resultados no pueden ser generalizables. Esta deficiencia resulta difícil de subsanar dado que, hasta la fecha, no se cuenta 
con mediciones gubernamentales oficiales que den cuenta sobre tal población, por tanto, no es posible generar muestras probabilísticas. Es el deseo de quienes escriben abordar esta situación en un futuro, con el trabajo conjunto entre la UNMDP y el gobierno de la ciudad. De forma complementaria, se espera avanzar con el rol de la educación superior en desarrollo emprendedor, analizando las competencias que fomentan las universidades para emprender, las diferencias motivacionales entre estudiantes varones y mujeres, y en la intervención estratégica de la educación superior en el fortalecimiento de mujeres emprendedoras.

\section{Referencias}

Alderfer, C. P. (1972). Existence, relatedness, and growth: human needs in organizational settings. Nueva York: Free Press.

Bandura, A. (2001). Guía para la construcción de escalas de autoeficacia. Recuperado de https://bit.ly/2TuqbB7

Bargsted, A. (2013). El emprendimiento social desde una mirada psicosocial. Civilizar: Ciencias Sociales y Humanas, 13(25), 121132. DOI: https://doi.org/10.22518/16578953.133

Bolaños, R. (2006). Impacto del modelo a imitar en la intención de crear una empresa. Estudios de Economía Aplicada, 24(2), 491-508.

Caamaño, E. (2010). Mujer y trabajo: origen y ocaso del modelo del padre proveedor y la madre cuidadora. Revista de Derecho (Valparaíso), (34), 179-209.

Cataño, L. R.; Tamanini, M. (2015). Mujeres emprendedoras en Colombia: una cuestión de género. XI Jornadas de Sociología. Facultad de Ciencias Sociales, Universidad de Buenos Aires, Argentina, 13-17 de julio.

Cedeño, J. (2013). Modelo de emprendedurismo para las mujeres profesionales en comunicación que desean desarrollar una marca personal (Tesis de maestría). Universidad Estatal a Distancia, Costa Rica. Recuperado de https://bit.ly/34B0yFg

Da Fonseca, B. M. da; Moriano, J. A.; Laguía, A.; Salazar, V. J. (2015). El perfil psicosocial del emprendedor: un estudio desde la perspectiva de género. Anuario de Psicología, 45(3), 301-315.

Díaz, J. C.; Hernández, R. M.; Barata, M. L. (2007). Estudiantes universitarios y creación de empresas. Un análisis comparativo entre España y Portugal. En J. C. Ayala (Coord.) Conocimiento, innovación y emprendedores: camino al futuro. (1338-1355). Madrid: Grupo Fedra.

Drucker, P. F. (2002). The discipline of innovation. Harvard Business Review, 80, 95-104.

Durán, E. (2013). Distinción entre actitud emprendedora y autoeficacia emprendedora: validez y confiabilidad en estudiantes universitarios. Educación y Futuro Digital, 5(7), 59-69.
Durán, E.; Arias, D. (2015). Intención emprendedora en estudiantes universitarios: integración de factores cognitivos y socio-personales. Revista Colombiana de Ciencias Sociales, 6(2), 320-340. DOI: https://doi.org/10.21501/22161201.1528

Dutra, A. I.; Rossi, A. S. (2011). Emprendedurismo en Uruguay (Tesis de grado). Universidad de la República. Montevideo, Uruguay. Recuperado de https://bit.ly/3mmFyrE

Fischel, A. (2013). ¿Cómo educar en emprendedurismo social $y$ ética? Ponencia presentada en el Congreso Internacional Emprendedurismo Social. Universidad de Costa Rica, Montes de Oca, Costa Rica, 6-7 de junio.

García, B. J.; Espino, L. E. (2013). La mujer emprendedora mexicana. Boletín Científico de las Ciencias Económico Administrativas del ICEA, 1(2). DOI: https://doi. org/10.29057/icea.v1i2.44

García, F. J. F.; Cañizares, S. M. S. (2010). Análisis del perfil emprendedor: una perspectiva de género. Estudios de Economía Aplicada, 28(3), 1-28.

García, E. G.; Ordoñez, L. O.; Avilés, E. A. (2016). Motivaciones y obstáculos para el emprendedurismo: una perspectiva de género desde los jóvenes universitarios. Retos. Revista de Ciencias de la Administración y Economía, 6(11), 43-52. DOI: https://doi.org/10.17163/ret.n11.2016.03

Garfinkel, H. (1991). Studies in etnomethodology. Nueva York: Prentice Hall.

GEM. (2016). Global Entrepreneurship Monitor. Recuperado de https://www.gemconsortium.org

Guerrero, L.; Canibe, F.; Armenteros, M.; López, S.; Reyna, G. (2012). Construcción de un perfil de las mujeres emprendedoras en Torreón, Coahuila, México. Global Conference on Business and Finance Proceedings, 7(2), 15961602.

Guzmán, A.; Trujillo, M. A. (2008). Emprendimiento social revisión de literatura. Estudios Gerenciales, 24(109), 105-125. DOI: https://doi.org/10.1016/S0123-5923(08)70055-X 
Heller, L. (2010). Mujeres emprendedoras en América Latina y el Caribe: realidades, obstáculos y desafíos. Santiago de Chile: Naciones Unidas, Cepal.

Henrekson, M. (2007). Entrepreneurship and Institutions. Labor Law \& Policy Journal, 28(3), 717-742. DOI: https://doi. org/10.2139/ssrn.996807

Hisrich, R. P. (2008). Entrepreneurship. Nueva York: McGraw Hill-Irwin.

Jirón, E.; Chan, H. G. (2016). Jornadas emprendedoras: generando espacios para ideas emprendedoras de la población estudiantil de UNED. Revista Electrónica Calidad en la Educación Superior, 7(2), 203-230. DOI: https://doi. org/10.22458/caes.v7i2.1607

Kanter, R. (1977). Men and women in corporation. Nueva York: Basic Books.

Karimi, S.; Biemans, H. J. A.; Lans, T.; Chizari, M.; Mulder, M. (2014). Effects of role models and gender on students' entrepreneurial intentions. European Journal of Training and Development, 38(8), 694-727.DOI: https://doi.org/10.1108/ EJTD-03-2013-0036

Kirzner, I. (1973). Competition and entrepreneurship. Londres: The University of Chicago Press.

Knight, F. (1947). Riesgo, incertidumbre y beneficio. Madrid: Aguilar.

Langowitz, N.; Minniti, M. (2007). The entrepreneurial propensity of women. Entrepreneurship Theory and Practice, 31(3), 341-364. DOI: https://doi.org/10.1111\%2Fj.15406520.2007.00177.x

Loli, A.; Dextre, E., Carpio, J. del; La Jara, E. (2010). Actitudes de creatividad y emprendimiento en estudiantes de la Universidad Nacional de Ingeniería y su relación con algunas variables socio demográficas. Revista de Investigación en Psicología, 13(2), 139-151. DOI: https://doi.org/10.15381/ rinvp.v13i2.3722

López, A. (2013). Emprender: una perspectiva de género. Coruña, España: Universidade da Coruña Servizo de Publicacións, Consorcio Editorial Galego.

López, M.; Romero, P.; Díaz, R. (2012). Motivaciones para emprender: un análisis de diferencias entre hombres y mujeres. Ei, 383, $75-84$.

López, Á.; Vázquez, P.; Montes, C. (2010). Mobbing: antecedentes psicosociales y consecuencias sobre la satisfacción laboral. Revista Latinoamericana de Psicología, 42(2), 215-224.

Martínez, F. M.; Carmona, G. (2009). Aproximación al concepto de «competencias emprendedoras»: valor social e implicaciones educativas. REICE. Revista Iberoamericana sobre Calidad, Eficacia y Cambio en Educación, 7(3) 82-98.

Maslow, A. (1954). Motivation and personality. Nueva York: Harper \& Row.
McClelland, D. C. (1961). The achieving society. Princeton: D. Van Nostrand Company. DOI: https://doi.org/10.1037/14359-000

Mejía, J. (2000). El muestreo en la investigación cualitativa. Investigaciones Sociales, 4(5), 165-180. DOI: https://doi. org/10.15381/is.v4i5.6851

Merino, M.; Vargas, D. (2011). Evaluación comparativa del potencial emprendedor de Latinoamérica: una perspectiva multinivel. Academia. Revista Latinoamericana de Administración, 3(46), 38-54.

Molero, F.; Recio, P.; Cuadrado, I. (2010). Liderazgo transformacional y transaccional: un análisis de la estructura factorial del multifactor Leardership Questionaire (MLQ). Psicothema, 22(3), 495-501.

Narváez, M. J. (2012). Dimensiones del emprendedurismo desde una visión universitaria. Ing-novacion, 2(4) 1-7.

Oliveira, B. M.; Moriano, J. A.; Laguía, A.; Salazar, V. J. (2015). El perfil psicosocial del emprendedor: un estudio desde la perspectiva de género. The UB Journal of psychology, 45(3), 301-315.

Ormeño, B. O. (2014). Emprendimiento femenino: propuesta de un perfil en base al propio discurso de mujeres, desde una perspectiva cualitativa. Gestión de las Personas y Tecnología, 7(19), 20-25.

Ornelas, C. E. C.; Contreras, L.; Silva, D. M.; Liquidano, M. D. C. (2015). El espíritu emprendedor y un factor que influencia su desarrollo temprano. Conciencia Tecnológica, (49), 46-51.

Ortiz, M.; Moncayo. B.; Riaño, A. (2010). Caracterización de las dimensiones del liderazgo transformacional en estudiantes de pregrado de la Universidad Militar Nueva Granada. Revista Educación y Desarrollo Social 4(2), 60-74.

Palacios, G. (2010). Emprendimiento social: integrando a los excluidos en el ámbito rural. Revista de Ciencias Sociales, 16(4), 579-590.

Pino, R.; Aguilar, M. Á. (2013). La inteligencia emocional como una herramienta de la gestión educativa para el liderazgo estudiantil. Cuadernos de Administración, 29(50), 132-141. DOI: https://doi.org/10.25100/cdea.v29i50.49

Pizarro, O; Amorós, J. E.; (2006). Mujeres y dinámica emprendedora: un estudio exploratorio. Universidad \& Empresa, 5(11) 15-32.

Rábago, P. L.; D’ Annunzio, M. C.; Monserrat, S. (2004). El perfil de mujeres emprendedoras exitosas de Argentina. II CIPEAL Conferencia Internacional de Pesquisa em Emprendedorismo na America Latina. Río de Janeiro, Brasil.

Ramos, A.; Barberá, E.; Sarrió, M. (2003). Mujeres directivas, espacio de poder y relaciones de género. Anuario de Psicología, 34(2), 267-278.

Roberts, D.; Woods, C. (2005). Changing the world on a shoestring: the concept of social entrepreneurship. University of Auckland. Business Review, 7(1) 45-51. 
Rodríguez, C. A.; Prieto, F. A. (2009). La sensibilidad al emprendimiento en los estudiantes universitarios. Estudio comparativo Colombia-Francia. Innovar. Revista de Ciencias Administrativas y Sociales, 19, 73-89.

Rompato, M. E. (2018). Emprendedurismo en la Argentina del siglo XXI. V Jornadas SIMEL (Sistema de Información del Mercado Laboral). Facultad de Humanidades, Universidad Nacional de Mar del Plata, Argentina, 20-21 de septiembre.

Rompato, M. E.; Silva, Y. F.; Zucarelli, M. (2018). ¿El becario de investigación es un emprendedor universitario? Un análisis exploratorio. VIII Congreso Marplatense de Psicología. Facultad de Psicología, Universidad Nacional de Mar del Plata, Argentina, 29 de noviembre-01 de diciembre.

Saavedra, M. L.; Camarena, M. E. (2015). Retos para el emprendimiento femenino en América Latina. Criterio Libre, 13(22), 129-152. DOI: https://doi.org/10.18041/19000642/criteriolibre.2015v13n22.129

Salazar, P. F.; Herrera, I. M.; Rueda, S.; León, J. M. (2014). El efecto de la conservación de recursos sobre la intención emprendedora en el contexto de crisis económica: el rol moderador de la autoeficacia y la creatividad. Anales de Psicología, 30(2), 549-559. DOI: https://doi.org/10.6018/ analesps.30.2.159281

Schumpeter, J. (1935). Análisis del cambio económico. Ensayos sobre el ciclo económico. México: Fondo de Cultura Económica.

Schwartz, S. H. (1996). Value priorities and behaviour: applying a theory of integrated value systems. En C. Seligman; J. M. Olson; y M. P. Zanna (Eds.) The psychology of values: The Ontario Symposium. (Vol. 8, 1-24). Mahwah, NJ: Lawrence Erlbaum.

Schwartz, S. H. (2005). Robustness and fruitfulness of a theory of universals in individual human values. En A. Tamayo; J. B. Porto (Eds.) Valores e comportamento nas organizacões. Petrópolis, Brasil: Vozes.
Siles, J.; Solano, C. (2007) Estructuras sociales, división sexual del trabajo y enfoques metodológicos: la estructura familiar y la función socio-sanitaria de la mujer. Investigación y Educación en Enfermería, 25(1), 66-73.

Silva, T. E. (2007). Movimiento empresa junior. El emprendedurismo. Revista Enfoque, (4), 4-7.

Soto, M.; Acevedo, A.; Labrador, L. (2015). La neuroinnovación del ser característica potenciadora del emprendimiento social. Cultura, Educación y Sociedad, 6(2), 51-62.

Traverso, J.; Irribarren, L. A.; Román, M. (2014). Emprendimiento femenino y desempeño de las empresas en la República de Chile. $V$ Congreso Universitario Internacional Investigación y Género. SIEMUS (Seminario Interdisciplinar de Estudios de las Mujeres de la Universidad de Sevilla), Universidad de Sevilla, Sevilla, España, 3-4 de julio.

Tobío, C. (2012). Cuidado e identidad de género. De las madres que trabajan a los hombres que cuidan. Revista Internacional de Sociología, 70(2), 399-422. DOI: https://doi.org/10.3989/ ris.2010.08.26

Veciana, J. M.; Aponte, M.; Urbano, D. (2005). University students' attitudes towards entrepreneurship: a two countries comparison. The International Entrepreneurship and Management Journal, 1(2), 165-182. DOI: https://doi. org/10.1007/s11365-005-1127-5

Villa, A.; Poblete, M. (2008). Aprendizaje basado en competencias. Una propuesta para la evaluación de competencias genéricas. Madrid: Ed. Mensajero.

Wilson, F.; Kickul, J.; Marlino, D. (2007). Gender, entrepreneurial self-efficacy, and entrepreneurial career intentions: implications for entrepreneurship Education 1. Entrepreneurship theory and practice, 31(3), 387-406. DOI: https://doi.org/10.1111/j.1540-6520.2007.00179.x 